Article

\title{
Map-Based Power-Split Strategy Design with Predictive Performance Optimization for Parallel Hybrid Electric Vehicles
}

\author{
Jixiang Fan ${ }^{1,2}$, Jiangyan Zhang ${ }^{1,3}$ and Tielong Shen ${ }^{2, *}$ \\ ${ }^{1}$ Department of Engineering and Applied Science, Sophia University, Tokyo 102-8554, Japan; \\ E-Mails: hwfjx@163.com (J.F.); zhangjy@ sophia.ac.jp (J.Z.) \\ ${ }^{2}$ Department of Electronic Engineering, Harbin Normal University, Harbin 150025, China \\ ${ }^{3}$ College of Electromechanical \& Information Engineering, Dalian Nationalities University, \\ Dalian 116600, China \\ * Author to whom correspondence should be addressed; E-Mail: tetu-sin@ sophia.ac.jp; \\ Tel.: +81-30-3238-3306.
}

Academic Editor: Enrico Sciubba

Received: 21 July 2015 / Accepted: 31 August 2015 / Published: 14 September 2015

\begin{abstract}
In this paper, a map-based optimal energy management strategy is proposed to improve the consumption economy of a plug-in parallel hybrid electric vehicle. In the design of the maps, which provide both the torque split between engine and motor and the gear shift, not only the current vehicle speed and power demand, but also the optimality based on the predicted trajectory of vehicle dynamics are considered. To seek the optimality, the equivalent consumption, which trades off the fuel and electricity usages, is chosen as the cost function. Moreover, in order to decrease the model errors in the process of optimization conducted in the discrete time domain, the variational integrator is employed to calculate the evolution of the vehicle dynamics. To evaluate the proposed energy management strategy, the simulation results performed on a professional GT-Suit simulator are demonstrated and the comparison to a real-time optimization method is also given to show the advantage of the proposed off-line optimization approach.
\end{abstract}

Keywords: hybrid electric vehicle; energy management; power split; variational integrator 


\section{Introduction}

In recent decades, the issues of environmental pollution and energy shortage have greatly promoted the development of new generation vehicles, such as electric vehicles (EV), fuel cell electric vehicles (FCEV) and hybrid electric vehicles (HEV). Although the EV and FCEV possess the most environmental superiority in the future, the short run range and high battery price restrict its short-term application. While the HEV has a long operation mileage and relatively low cost, it offers the best promise in the short to mid term [1]. Especially, the plug-in HEV (PHEV) [2] is more competitive than the conventional HEV [3], because it can make full use of the inexpensive electricity from the grid [4].

The power management strategy is one of the most effective ways to exploit the potential of HEV in fuel economy and emission reduction. A number of strategies, some of them usually applicable to both plug-in and conventional HEVs, have been proposed, as summarized and compared by Chua [5], Wirasingha [6] and Serrao [7]. These strategies mainly share a common target, namely to split the power/torque requirement in multiple power sources to achieve the best performance. The early surveys concentrated on the development of the rule- or logic-based scheme [8-13]. These rules, in general, are constructed according to the engineering expertise and insight. The main idea of this type of control strategy is to make each actuator operate as close to optimality as possible at an instant time point. Although these control strategies are simple and easy to implement, they are generally sub-optimal; in other words, they cannot theoretically guarantee optimality; hence, they cannot adequately exploit the potential advantages of $\mathrm{HEV}$.

The optimization-based method, as a natural extension of earlier methods, has attracted increasing attention [14-17]. One of the powerful optimization-based methods is dynamical programming (DP), which has illustrated the effectiveness in optimal energy management control for HEVs [18-22]. As a global optimization method, DP is successful in deciding the optimal power management strategy for a given drive cycle or a probabilistic distribution of drive cycles; the result of DP thus is usually used as a benchmark for assessing other strategies [23]. However, this technique is clearly infeasible for real-time control due to the full knowledge of a drive cycle not being able to be known a priori. For tackling the challenge on the non-causality of the DP algorithm, the predictive control methods, the model predictive control (MPC) being a typical one, were employed to realize causal and dynamic optimization control in power management of HEVs [24,25]. The MPC algorithm achieves the on-line optimization by predicting the future driving pattern in a finite receding horizon according to the vehicle's current states and input variables (for example, vehicle speed, battery SOC, gear number, power/torque demand, etc.) [26-28]. However the complexity of the algorithm results in considerable computational load, which creates a barrier for real-time on-line solutions and applications. Solving the complex predictive control problem offline and storing the solution in look-up tables for on-line use thus becomes an effective means to reduce the burden of the processor [29,30]. In recent years, developing a fast algorithm to save the computation time of on-line optimization has been attracting more attention $[31,32]$.

This paper primarily focuses on the power-split problem under the hybrid mode of PHEVs. A pair of static maps, which are designed by the optimal approach and solved offline, are used as the set of decisions for the extraction of real-time control inputs. This method can significantly reduce 
the computational burden on the premise of ensuring local optimality. In the design of maps, the variational integrator, which preserves the geometric structures or properties of the numerical flow of the mechanical system, is used to discretize the vehicle dynamics. This discrete method produces an improved qualitative behavior for the vehicle dynamics; the obtained optimal solution therefore is more accurate than general-purpose methods under the same time step.

The rest of this paper is arranged as follows. Section 2 presents the HEV architecture and describes the energy management problem of PHEVs. Section 3 designs the optimization index for the PHEV power-split problem, sets up the discrete models for the energy converters (engine, EM and battery) and vehicle and presents the numerical optimization algorithm. Section 4 compares our results with an on-line solution strategy and discusses the results in various weighting factors. Finally, the conclusions are given in Section 5.

\section{Problem Description}

\subsection{Architecture}

Different from the architectures of the Chevrolet Volt [2] and Toyota Prius [3], in which planetary gear sets are used, in this study, the architecture of a plug-in parallel HEV with a five-speed gearbox is considered and illustrated in Figure 1. As can be seen, this HEV system includes two power sources: internal combustion engine (ICE) and electric machine (EM). The ICE is connected to the EM shaft through Clutch I; the EM is connected to real wheels through Clutch II and the five-speed gearbox. The parallel HEV system mainly works under the one of following three modes: (1) EV mode, when only Clutch II is engaged; (2) battery recover (BR) mode, when only Clutch I is engaged; and (3) HEV mode, when Clutches I and II are engaged and the engine and motor work together. Specifically, the EM integrates the starter, generator and motor; energy regeneration is available for this architecture.

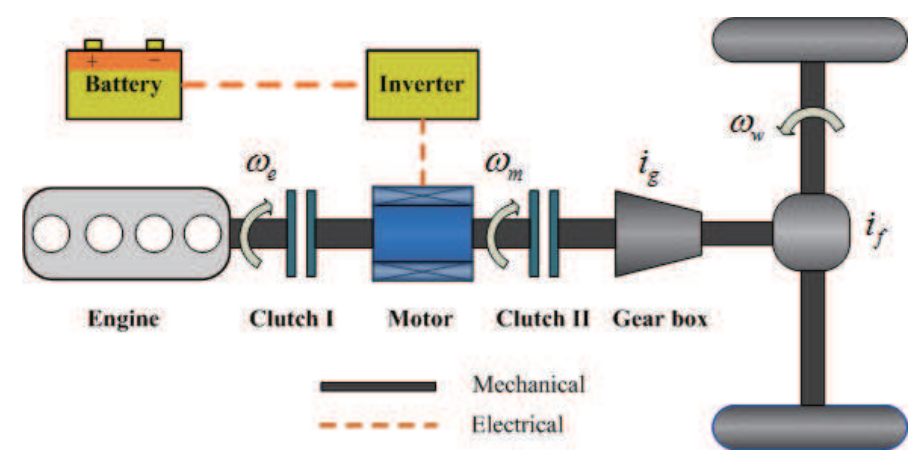

Figure 1. Architecture of the parallel hybrid electric vehicle.

\subsection{Control System Structure}

Figure 2 gives the schematic diagram of the vehicle control system. As shown in this figure, the control system of the PHEV consists of two major parts, the mode select block and the energy management block. The inputs of the mode select block are the driver's intention (i.e., driving or braking) and the vehicle states (i.e., the vehicle speed and the battery SOC). The outputs of the mode select 
block are the mode switching commands and the instant power demand, which interprets the driver's accelerator or brake pedal position. Depending on these outputs, one of three different modes, i.e., EV mode, BR mode and HEV mode, is activated. The energy management block then outputs corresponding commands of the engine torque, motor torque and gear number to the powertrain and transmission. In the mode select part, the conditions for selecting different modes can be briefly explained as follows: If the battery SOC is below the maximum level and the vehicle is braking or the battery SOC is beyond the minimum level and the vehicle is starting, the EV mode is selected. If the SOC is too low, the mode select block will activates the BR mode. For normal driving circumstances, the HEV mode will be utilized.

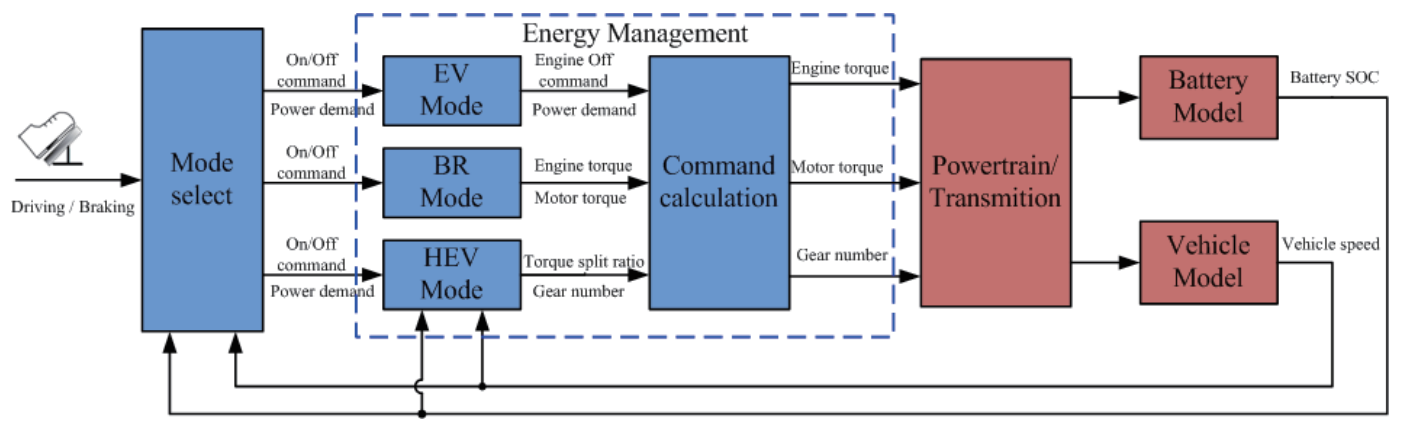

Figure 2. Schematic diagram of the vehicle control system.

In the EV mode, all power demand will be distributed to the motor during starting for the improvement of the fuel economy. During braking, the electric machine will act as a regenerator to recuperate all brake energy; unless it exceeds the capability of the electric powertrain, the rest of the energy, except for that regenerated by electric powertrain, will be absorbed by the hydraulic brake system. In the BR mode, the engine will operate in high efficient points to drag the regenerator for charging the battery; thereby, the energy conversion from fuel energy to electric energy is realized efficiently. In the HEV mode, the engine and motor will drive the vehicle cooperatively according to an appropriate power-split strategy, which distributes the torque between the motor and engine and chooses the gear number. Since in the EV and BR modes, some simple rule-based approaches can be employed to achieve power management, therefore in the current study, we will pay attention to developing the power-split strategy in the HEV mode.

\subsection{Power-Split Strategy in the HEV Mode}

In order to reduce the occupation of processor resources, in the HEV mode, the map-based energy management strategy is researched to realize the power distribution between engine and motor. The power-split maps, which consist of the torque distribution map and the gear select map, are created off-line based on the optimal method given in Section 3 in detail. The vehicle control system then extracts the decision from these maps in real time.

Figure 3 presents the schematic diagram of the power-split strategy. Based on instant power demand $P_{\text {dem }}$ and vehicle speed $v$, the power distribution between the engine and motor is achieved by making appropriate decisions, i.e., torque-split ratio $\lambda_{\tau}$ and gear ratio $i_{g}$, from two given maps. Then, the torque-split ratio is converted to the corresponding demand torques of the engine and motor by the 
command calculation, and the gear ratio is converted to the discrete gear number by a piecewise converting table, as listed in Table 1.

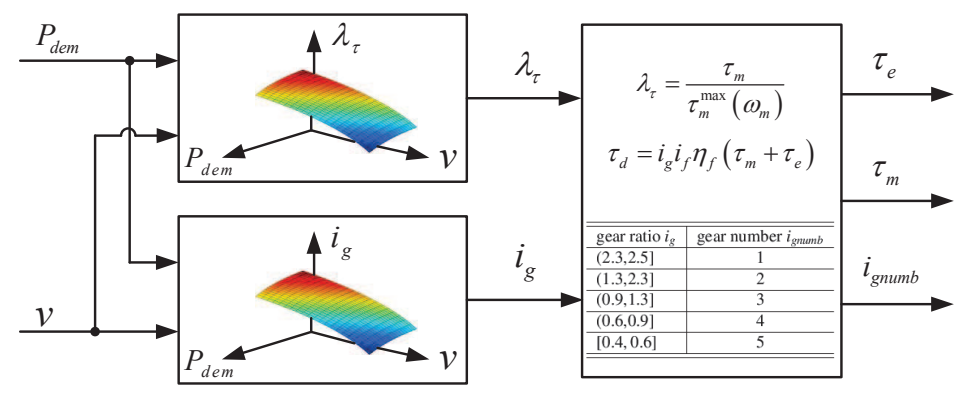

Figure 3. Control schematic diagram in the HEV mode.

Table 1. Converting table from the gear ratio to the gear number.

\begin{tabular}{cc}
\hline Gear Ratio $i_{g}$ & Gear Number $i_{\text {gnumb }}$ \\
\hline$(2.3,2.5]$ & 1 \\
$(1.3,2.3]$ & 2 \\
$(0.9,1.3]$ & 3 \\
$(0.6,0.9]$ & 4 \\
{$[0.4,0.6]$} & 5 \\
\hline
\end{tabular}

As a typical dual-power-source system, the PHEV complies with the power conservation, which can be regarded as an essential constraint and is given by Equation (1). The power conservation constraint ensures that the sum of power provided by the engine and motor is equal to the driver's power demand.

$$
P_{\text {dem }}=P_{e}+P_{m}
$$

where $P_{d e m}$ represents the driver's power demand and $P_{e}$ and $P_{m}$ represent the engine and motor power, respectively.

For the presented PHEV configuration, if neglecting the slipping dynamics of clutches and the torsion damping of the transmission system, and considering the coaxial characteristic of the engine and motor, the rotational speeds of the wheel axle, engine and motor are subject to the following equality constraint:

$$
i_{g} i_{f} \omega_{w}=\omega_{m}=\omega_{e}
$$

where $i_{g}$ and $i_{f}$ represent the gear ratios of the transmission and final gear, respectively. $\omega_{w}, \omega_{m}$ and $\omega_{e}$ represent the rotational speeds of the wheel axle, engine and motor, respectively.

Based on the power formula:

$$
P_{d e m}=\tau_{d} \omega_{w}
$$

where $\tau_{d}=\tau_{\text {drive }}-\tau_{\text {brake }}, \tau_{\text {drive }}$ represents the traction torque, $\tau_{\text {brake }}$ represents the friction brake torque. The drive's power demand thus can be transformed to torque demand according to the current vehicle speed $v$ and the radius of wheel $R_{w}$ with $v=\omega_{w} R_{w}$. 
Taking into account the fact that the output power of each actuator of the PHEV is equal to the product of its angular velocity and output torque, therefore at the wheel axle, the following torque balance equation can be obtained by substituting Equation (2) into Equation (1).

$$
\tau_{d}=i_{g} i_{f}\left(\tau_{m}+\tau_{e}\right)
$$

where $\tau_{e}$ and $\tau_{m}$ represent the torques distributed to the engine and motor, respectively.

Considering the limitation of maximum torque of motor, the torque-split ratio $\lambda_{\tau}$ is defined as:

$$
\lambda_{\tau}=\frac{\tau_{m}}{\tau_{m}^{\max }\left(\omega_{m}\right)}
$$

where $\tau_{m}^{\max }$ denotes the maximum output torque of motor. The corresponding torque distributed to the engine thereby can be calculated by torque balance Equation (4).

According to the power-split strategy described above, the design of two maps becomes the key issue for the energy management in the HEV mode; the detailed design method will be presented in the following section.

\section{Design of Power-Split Control Maps}

\subsection{Optimal Power Split Formulation}

For the presented HEV system, it should be noted that at each sampling time, the decisions of $\lambda_{\tau}$ and $i_{g}$ affect not only the current states of the powertrain, but also the transient profile of the state during a period since the systems of the vehicle and battery are a dynamical system. Moreover, at the current time, simply noted as $t=0$, the decisions of $\lambda_{\tau}$ and $i_{g}$ according to the current states (vehicle speed $v(0)$ and demand power $P_{d e m}(0)$ ) influence not only the fuel and electricity consumption at the time $t=0$, which can be described by the fuel mass flow rate $\dot{m}_{f}(0)$ and the instantaneous change rate of the energy stored in battery $\dot{m}_{e}(0)$, but also $\dot{m}_{f}(t)$ and $\dot{m}_{e}(t), t \subset[0, T]$, where $T$ defines a given finite time horizon. Since vehicle speed $v(t), t \subset[0, T]$ is determined by the vehicle dynamics forced by the demand power $P_{d e m}$. Therefore, for the decisions of $\lambda_{\tau}$ and $i_{g}$, the following function is introduced as the cost function.

$$
J\left(\lambda_{\tau}, i_{g}\right)=\int_{0}^{T}\left[\zeta \alpha_{f} \dot{m}_{f}\left(v, \lambda_{\tau}, i_{g}, P_{d e m}, t\right)+\dot{m}_{e}\left(v, \lambda_{\tau}, i_{g}, t\right)\right] d t
$$

subject to:

$$
\dot{v}=f\left(v, P_{d e m}, \theta\right), v(0)=v_{0}, P_{d e m}(0)=P_{0}
$$

where $\theta$ is the road slope.

The maps to be designed hence provide the optimal decision $u^{*}=\left[\lambda_{\tau}^{*}, i_{g}^{*}\right]^{T}$ as:

$$
\left[\begin{array}{c}
\lambda_{\tau}^{*} \\
i_{g}^{*}
\end{array}\right]=\arg \min _{u \in U} J\left(\lambda_{\tau}, i_{g}\right)
$$

according to each possible current state $\left(v_{0}, P_{0}\right)$.

Since the cost function expressed by Equation (6) is the integral of the fuel and electricity consumption during a predictive time horizon $T$, the decision drawn from the maps thus will make the proposed performance, i.e., the fuel and electricity consumption, optimal in a predictive time horizon $T$. 
In the cost function Equation (6), $\dot{m}_{f}$ represents the fuel consumption rate in grams per unit time; the coefficient $\alpha_{f}$ with the expression $\alpha_{f}=Q_{l h v} \times 1000$ converts it to the energy consumption rate in watts. $Q_{l h v}$ is the fuel lower heating value. $\dot{m}_{e}$ represents the electricity consumption rate in watts and can be calculated by the dynamics of the battery SOC as follows:

$$
\dot{m}_{e}=-U_{o c} Q_{b a t t} S \dot{O} C
$$

It is clear that the $\dot{m}_{e}$ is positive when the PHEV consumes the electric energy stored in the battery and negative during regeneration. The parameter $\zeta$, which represents the relative gasoline to grid electricity price per joule, is defined as the energy price ratio and calculated by:

$$
\zeta=\frac{\frac{\gamma_{f}}{\rho_{f} Q_{l h v}} \times 1000}{\frac{\gamma_{e}}{1000 \times 3600}}
$$

where $\gamma_{f}$ and $\gamma_{e}$ are the prices of gasoline per liter and grid electricity per kilo-watt hour, respectively. $\rho_{f}$ is the fuel density of gasoline in grams per liter.

As can be observed in cost function Equation (6), the first term in the integral represents the fuel consumption rate, and the second one represents the electricity consumption rate. The parameter $\zeta$ is viewed as the weighting factor. Hence, the cost function can be comprehended as the weighted sum on the consumption of PHEV fuel and electricity. In fact, the cost function is equivalent to the cost of energy by multiplying the electricity price in this equation. Therefore, using the different value of the parameter $\zeta$, the tradeoff between the fuel and electricity cost can be evaluated.

Different from the conventional HEV, which needs to sustain the battery SOC at a specified value at the end of trip, the plug-in HEV as considered in this study is not intensively concerned about the terminal value of battery SOC after a trip. Hence, no terminal constraint on the SOC is imposed on the above problem formulation.

For the decision $u$, the corresponding physical constraints, including gear shift ratio and the torque split ratio constraints, need to be considered. The gear shift ratio must be subjected to the mechanical constraint between the maximum and minimum gear ratio of the transmission. The torque distributed to the motor is limited between the maximum and minimum torque provided by the motor. Therefore, the decision constraints can be formulated in the following admissible control set.

$$
U=\left\{u: \begin{array}{c}
i_{g}^{\min } \leq i_{g} \leq i_{g}^{\max } \\
-1 \leq \lambda_{\tau} \leq 1
\end{array}\right\}
$$

where $i_{g}^{\min }=0.4$ and $i_{g}^{\max }=2.5$ are the bounds of the transmission gear ratio.

In optimizing the power management, it is necessary to impose some practical constraints on the physical system to ensure the safe and smooth operation of the engine, motor and battery. Generally, these considered constraints, usually time-varying, can be written in mathematical form as:

$$
\begin{gathered}
\omega_{e}^{\min } \leq \omega_{e} \leq \omega_{e}^{\max }, \tau_{e}^{\min }\left(\omega_{e}\right) \leq \tau_{e} \leq \tau_{e}^{\max }\left(\omega_{e}\right) \\
\omega_{m}^{\min } \leq \omega_{m} \leq \omega_{m}^{\max }, \tau_{m}^{\min }\left(\omega_{m}\right) \leq \tau_{m} \leq \tau_{m}^{\max }\left(\omega_{m}\right) \\
S O C^{\min } \leq S O C \leq S O C^{\max }
\end{gathered}
$$


where ${ }^{\min }$ and ${ }^{\max }$ represent the corresponding minimum and maximum bounds. The battery SOC limitations, $S O C^{\mathrm{min}}$ and $S O C^{\mathrm{max}}$, are 0.4 and 0.9 , respectively.

Based on the above method that calculates the optimal decision according to the current vehicle speed and power demand, the following steps are used to create the decision maps. At first, in terms of the specification of a given vehicle powertrain, the ranges of the vehicle speed and power demand are estimated and discretized in an appropriate interval. For each pair of discrete power demand and vehicle speed, the optimization algorithm then calculates the optimal control sequence in the finite time horizon. Moreover, the first elements in the optimal solution sequences of the gear ratio and torque split ratio are extracted as a pair of decisions. Finally, for different vehicle speed and power demand, a series of different decision pairs are obtained, and the corresponding maps are drawn by the curve fitting method.

\subsection{Discrete Vehicle Model}

Since the vehicle dynamics is the constraint in solving the optimal power split problem of PHEV and the fuel and electricity consumption are closely related to the vehicle speed, therefore, the calculation precision of vehicle speed in each time horizon directly influences the precision of energy consumption, consequently influencing the accuracy of the decision. An accurate discretization method for the vehicle dynamics thus can significantly decrease the decision deviation. Consequently, in order to accurately describe the evolution of vehicle speed in the discrete time region, the discrete variational method, which does a direct discretization of the Lagrange-d'Alembert principle, is utilized to derive the discrete dynamics of the vehicle system.

For a forced Lagrange system, the Lagrange-d'Alembert principle is formulated as [33,34]:

$$
\delta \int_{0}^{T} L(q(t), \dot{q}(t)) d t+\int_{0}^{T} f(t) \cdot \delta q(t) d t=0
$$

where $q(t)$ and $\dot{q}(t)$ are the generalized coordinate and velocity, respectively. $L$ is the Lagrangian; $f$ is the corresponding Lagrange force.

We replace the continuous path $q:[0, T] \rightarrow Q$ by a discrete path $q_{d}:\{0, h, \ldots, N h=T\} \rightarrow Q$, with a selected time step $h$ (in general, $h$ is regarded as the sampling period in real-time control), and write $q(k)=q_{d}(k h)$ as an approximation of $q(k h)$. Furthermore, we approximate the action functional (the first integral in Equation (15)) on a time step $[k h,(k+1) h]$ by a discrete Lagrangian $L_{d}$ :

$$
L_{d}(q(k), q(k+1)) \approx \int_{k h}^{(k+1) h} L(q(t), \dot{q}(t)) d t
$$

and the virtual work (the second integral in Equation (15)) by the expression of following form:

$$
f^{-}(k) \cdot \delta q(k)+f^{+}(k) \cdot \delta q(k+1) \approx \int_{k h}^{(k+1) h} f(t) \cdot \delta q(t) d t
$$

where $f^{-}(k)$ and $f^{+}(k)$ represent the left and right discrete Lagrange forces, respectively.

The discrete version of the Lagrange-d'Alembert principle can be obtained by summing the discrete Lagrangian and virtual work from $k=0$ to $k=N-1$, which is equivalent to the following forced discrete Euler-Lagrange equation.

$$
D_{2} L_{d}(q(k-1), q(k))+D_{1} L_{d}(q(k), q(k+1))+f^{+}(k-1)+f^{-}(k)=0
$$


where $D_{i}$ denotes the differentiation with respect to the $i$-th component and $k=1, \ldots, N-1$. In turn, Equation (18) gives rise to the forced discrete Lagrangian map:

$$
\Phi_{L_{d}}^{f}:(q(k-1), q(k)) \mapsto(q(k), q(k+1))
$$

In general, the Lagrange system described by states $(q, \dot{q})$ can be transformed to the Hamilton system described by states $(q, p)$ through Legendre transforms, where $p$ can be interpreted as the momentum. The discrete Lagrange system therefore can be transformed to a discrete Hamilton system by the forced discrete Legendre transforms:

$$
\begin{aligned}
& \mathbb{F}^{f-} L_{d} \quad: \quad(q(k), q(k+1)) \mapsto(q(k), p(k)) \\
& \mathbb{F}^{f+} L_{d} \quad: \quad(q(k), q(k+1)) \mapsto(q(k+1), p(k+1))
\end{aligned}
$$

with:

$$
\begin{gathered}
p(k)=-D_{1} L_{d}(q(k), q(k+1))-f^{-} \\
p(k+1)=D_{2} L_{d}(q(k), q(k+1))+f^{+}
\end{gathered}
$$

which gives the forced discrete Hamiltonian map:

$$
\tilde{\Phi}_{L_{d}}^{f}:(q(k), p(k)) \mapsto(q(k+1), p(k+1))
$$

with three steps evaluating formulation:

$$
\tilde{\Phi}_{L_{d}}^{f}=\mathbb{F}^{f-} L_{d} \circ \Phi_{L_{d}}^{f} \circ\left(\mathbb{F}^{f-} L_{d}\right)^{-1}
$$

As a classical mechanics system, the vehicle is considered to move on the road with the road slopes subjected to traction force. The Lagrangian of the vehicle system is the energy difference between the kinetic and potential energy as:

$$
L=T_{\text {vehicle }}-V_{\text {vehicle }}=\frac{1}{2} m \dot{x}^{2}-m g \sin \theta
$$

where $x$ is the vehicle displacement, $m$ is the vehicle mass and $g$ is the gravity acceleration.

The typical resistance loads applied to the vehicle include the rolling resistance of tires and the aerodynamics drag. These load models thus can be described by the following equations:

$$
\begin{gathered}
F_{\text {rolling }}=\mu_{r} m g \cos \theta \\
F_{\text {aero }}=\frac{1}{2} \rho A C_{d} v^{2}
\end{gathered}
$$

where $\mu_{r}$ is the rolling resistance coefficient, $\rho$ is the air density, $A$ is the frontal area of vehicle and $C_{d}$ is the aerodynamics drag coefficient.

Therefore, the Lagrange force is expressed by the following equation:

$$
f=\frac{\tau_{d}}{R_{w}}-F_{\text {rolling }}-F_{\text {aero }}
$$


In view of the computational efficiency and accuracy, the midpoint rule is utilized to discretize the Lagrangian and Lagrange force as:

$$
\begin{aligned}
L_{d}(q(k), q(k+1)) & =h \cdot L\left(\frac{q(k+1)+q(k)}{2}, \frac{q(k+1)-q(k)}{h}\right) \\
f^{ \pm}(k) & =\frac{h}{4}(f(k)+f(k+1))
\end{aligned}
$$

Derived from the three-step method given by Formulation (25) with corresponding Equations (18) to (23), the discrete dynamics of vehicle system can be obtained as:

$$
\begin{aligned}
x(k+1)= & x(k)+h v(k)-\frac{h^{2}}{2}\left(g \sin \theta+\mu_{r} g \cos \theta\right) \\
& +\frac{h^{2}}{4 m}\left[\frac{\tau_{d}(k)+\tau_{d}(k+1)}{R_{w}}-\frac{1}{2} \rho A C_{d}\left(v^{2}(k)+v^{2}(k+1)\right)\right] \\
v(k+1)= & v(k)-h\left(g \sin \theta+\mu_{r} g \cos \theta\right) \\
& +\frac{h}{2 m}\left[\frac{\tau_{d}(k)+\tau_{d}(k+1)}{R_{w}}-\frac{1}{2} \rho A C_{d}\left(v^{2}(k)+v^{2}(k+1)\right)\right]
\end{aligned}
$$

\subsection{Fuel and Electricity Consumption Model}

The ICE model is developed by focusing on the characteristics of fuel consumption and torque output. Ignoring the transient dynamics of the engine, the quasi-static model is introduced to the gasoline engine [35]. The fuel consumption of the engine can be calculated by using the engine efficiency extracted from the break specific fuel consumption (BSFC) map (shown in Figure 4), and subsequently, the following equivalent function with respect to the speed and torque of engine is employed to approximate the fuel consumption characteristic of the ICE.

$$
\begin{aligned}
\dot{m}_{f}(k) & =\mathcal{M} \mathcal{A P}\left(\omega_{e}(k), \tau_{e}(k)\right) \\
& =\tau_{e}(k) \sum_{i=1}^{4} b_{1 i} \omega_{e}^{i}(k)+\tau_{e}^{2}(k) \sum_{i=1}^{3} b_{2 i} \omega_{e}^{i}(k)+\tau_{e}^{3}(k) \sum_{i=1}^{2} b_{3 i} \omega_{e}^{i}(k)+b_{41} \tau_{e}^{4}(k) \omega_{e}(k \gamma 33)
\end{aligned}
$$

where $b_{11} \sim b_{14}, b_{21} \sim b_{23}, b_{31}, b_{32}$ and $b_{41}$ are polynomial coefficients.

According to the BSFC map, the following polynomial is obtained to characterize the maximum engine torque depending on the engine speed.

$$
\tau_{e}^{\max }\left(\omega_{e}(k)\right)=\sum_{i=1}^{6} b_{t i} \omega_{e}^{i}(k)
$$

where $b_{t 1} \sim b_{t 6}$ are polynomial coefficients. 


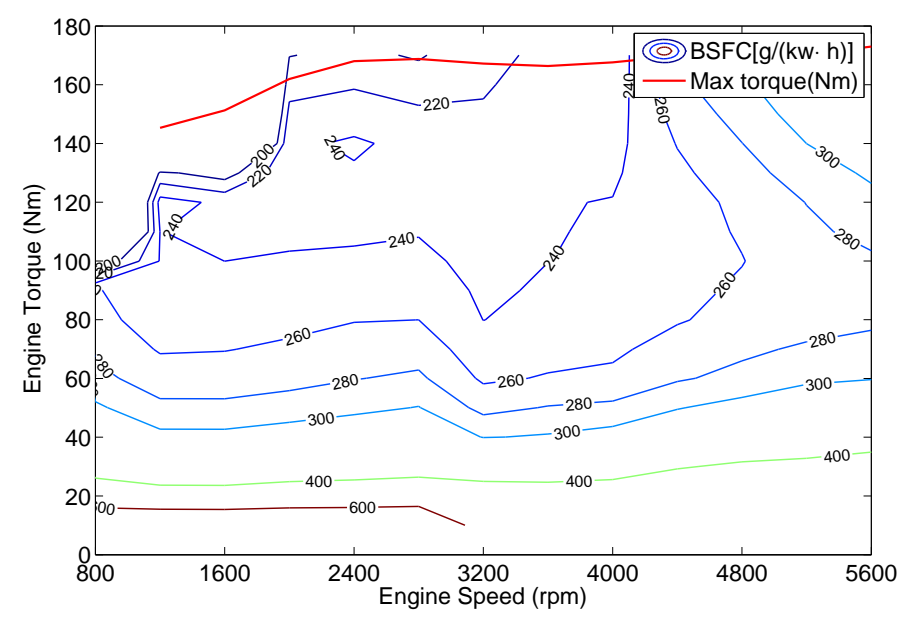

Figure 4. The fuel consumption efficiency map of ICE.

Based on the quasi-static assumption, the electric machine is modeled using the steady-state efficiency map [36], which can be expressed by the polynomial function with respect to the torque and speed of motor as:

$$
\begin{aligned}
\eta_{m}(k) & =\mathcal{F}\left(\tau_{m}(k), \omega_{m}(k)\right) \\
& =a_{0}+a_{1} \omega_{m}(k)+a_{2} \omega_{m}^{2}(k)+a_{3} \tau_{m}^{2}(k)+a_{4} \omega_{m}^{3}(k)+a_{5} \omega_{m}(k) \tau_{m}^{2}(k)
\end{aligned}
$$

where $a_{0} \sim a_{5}$ are polynomial coefficients. As the constraint of the motor operating points, the actual output torque of the motor is limited in the field between the maximum and minimum torques of the motor. These limitations are functions of the motor speed and are approximated by the following polynomial:

$$
\tau_{m}^{\max }\left(\omega_{m}(k)\right)=\sum_{i=0}^{5} a_{t i} \omega_{m}^{i}(k)
$$

where $a_{t 0} \sim a_{t 5}$ are polynomial coefficients.

The efficiency map and torque characteristic curve of the electric machine are given in Figure 5.

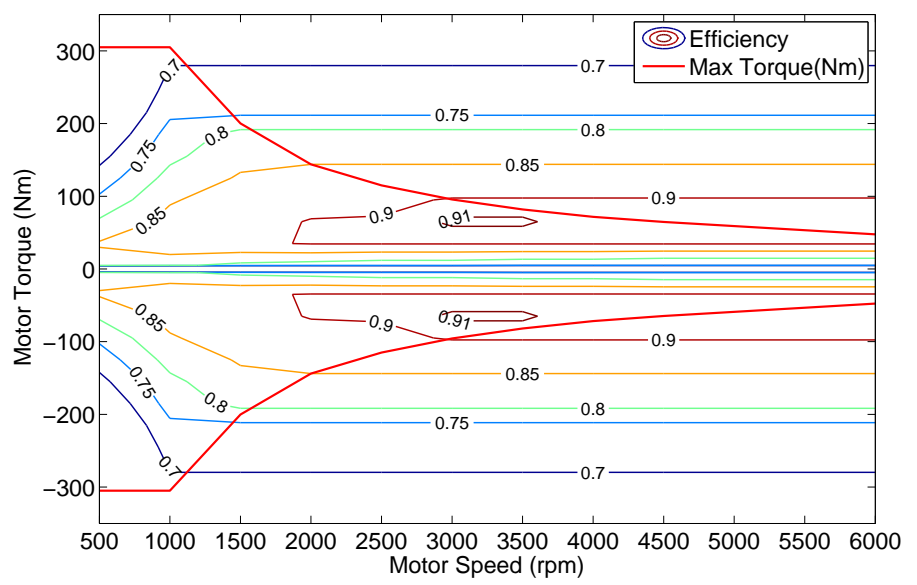

Figure 5. The electric machine (EM) efficiency map. 
In the electric drive system, the battery can be modeled as an ideal voltage source with open-circuit voltage $U_{o c}$ in series with a constant internal resistance $R_{\text {batt }}$ [19]. As important information on electrical power usage, the battery state-of-charge (SOC) depends on the battery current $I_{\text {batt }}$ and maximum battery charge capacity $Q_{b \max }$ according to the following dynamics of battery SOC:

$$
S \dot{O} C=-\frac{I_{b a t t}}{Q_{b \max }}
$$

The battery current $I_{b a t t}$ can be calculated from the battery power $P_{b a t t}$ by employing the following power balance relationship:

$$
P_{b a t t}=U_{o c} I_{b a t t}-R_{b a t t} I_{b a t t}^{2}
$$

By convention, positive battery power $\left(P_{\text {batt }}>0\right)$ indicates discharging, and negative battery power $\left(P_{\text {batt }}<0\right)$ indicates charging. Solving Equations (37) and (38), the dynamics of battery SOC can be rewritten as:

$$
S \dot{O} C(k)=-\frac{U_{o c}-\sqrt{U_{o c}^{2}-4 P_{b a t t}(k) R_{b a t t}}}{2 Q_{b \max } R_{b a t t}}
$$

Moreover, relating the battery power $P_{b a t t}$ to the speed, torque and efficiency of electric machine gives:

$$
P_{b a t t}(k)=\eta_{m}^{\lambda}(k) \tau_{m}(k) \omega_{m}(k)
$$

where $\lambda$ is the index with the following meaning:

$$
\lambda=\left\{\begin{array}{cc}
-1, & \tau_{m}(k) \omega_{m}(k)>0 \\
1, & \tau_{m}(k) \omega_{m}(k)<0
\end{array}\right.
$$

Combining Equation (39) to Equation (41) with the polynomial fitting Equation (35) of the motor efficiency presents a whole description of the dynamics of the battery SOC.

For solving the optimal control problem numerically, the cost function needs to be discretized besides the vehicle dynamics and consumption model. Considering the computational efficiency and accuracy, as well, the midpoint rule is utilized to discretize the cost function as:

$$
J\left(\lambda_{\tau}, i_{g}\right)=\sum_{k=0}^{N-1} h\left\{\frac{1}{2} \zeta \alpha_{f}\left[\dot{m}_{f}(k)+\dot{m}_{f}(k+1)\right]+\frac{1}{2}\left[\dot{m}_{e}(k)+\dot{m}_{e}(k+1)\right]\right\}
$$

and note that in Equation (42), the $\lambda_{\tau}$ and $i_{g}$ are discrete control sequences, i.e., $\lambda_{\tau}=\left[\lambda_{\tau}(0), \lambda_{\tau}(1), \cdots, \lambda_{\tau}(N-1)\right]$ and $i_{g}=\left[i_{g}(0), i_{g}(1), \cdots, i_{g}(N-1)\right]$.

Consequently, the optimal power split problem in discrete form can be described by discrete cost function Equation (42) and discrete vehicle dynamics constraint Equation (32), with control constraint set Equation (11) and some practical constraints Equation (12) to Equation (14).

\subsection{Optimization Algorithm}

In a time horizon $T$, the optimal power management problem is regarded as a constrained nonlinear optimization problem and solved by the nonlinear programming method. Since there exist nonlinear and inequality constraints, the optimal solution sequences on decisions $\lambda_{\tau}$ and $i_{g}$ are calculated based on 
a "sequential quadratic programming (SQP)" algorithm [37,38], which solves a sequence of optimization subproblems, each of which optimizes a quadratic model of the objective subject to a linearization of the constraints.

The implementation of SQP algorithm includes the following three main stages: (1) updating the hessian matrix; at each major iteration, a positive-definite quasi-Newton approximation of the hessian matrix of the Lagrangian is computed by employing the BFGS formula; (2) solving the quadratic programming (QP) subproblem; at each major iteration of the SQP method, a QP problem is solved by utilizing an active set strategy (also known as a projection method). The solution process consists of two phases. The first phase is the computation of a feasible point (if one exists). The second phase is the generation of an iterative sequence of feasible points that converge to the solution; (3) line search and merit function: the solution of the QP subproblem is utilized to produce a search direction, and then, a line search process is performed by utilizing a merit function.

In this study, suppose the road slope is constant zero and the power demand is invariable within a prediction horizon. We select the ranges of vehicle speed and power requirement as $0 \sim 100 \mathrm{~km} / \mathrm{h}$ and $0 \sim 70 \mathrm{~kW}$ with intervals of $4 \mathrm{~km} / \mathrm{h}$ and $5 \mathrm{~kW}$, respectively. The model is discretized with the time step of $1 \mathrm{~s}$, and the prediction horizon $T$ is selected as $8 \mathrm{~s}$. In order to estimate the impact of different relative prices of electricity to fuel on optimal power management, we select a constant fuel price of $\gamma_{f}=150$ JPY per liter and different electricity prices of $\gamma_{e}=20, \gamma_{e}=30$ and $\gamma_{e}=50$ JPY per kilo-watt hour; therefore, the corresponding energy price ratios are $\zeta=0.82, \zeta=0.55$ and $\zeta=0.33$, respectively. For each energy price ratio, two decision maps, one a gear number map, which can be converted from the solved gear ratio map, another a torque split ratio map, are presented in Figure 6 to Figure 8 and used in Section 4.

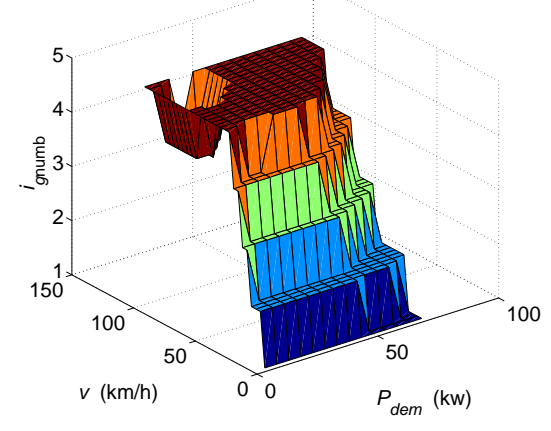

(a)

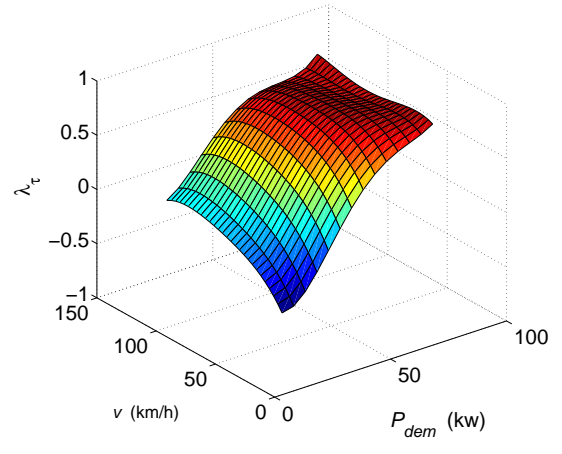

(b)

Figure 6. Decision maps, $\zeta=0.82$. (a) Gear number map; (b) torque split ratio map.

From the gear number maps in these three figures (i.e., Figures 6a,7a and 8a), it is noted that the gear numbers are increasing from one to five along with the increase of vehicle speed; this trend is consistent with the common gear selection strategy. However, in the present study, the selection of gear numbers does not uniquely depend on the vehicle; it also is affected by the power demand from the driver as described in the optimal power-split strategy above; hence, the gear number maps are not a normal ladder shape with straight edges. In some circumstances, jumps of the gear number exist at the edges of 
the ladder shape, especially for a relatively high power requirement; for example the power requirement is larger than $50 \mathrm{~kW}$, and the gear number is one lower than that in low power requirement; therefore, a ladder shape curve with approximate arc edges is formed in these maps.

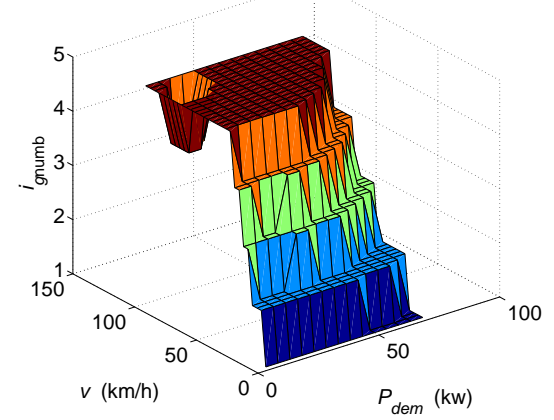

(a)

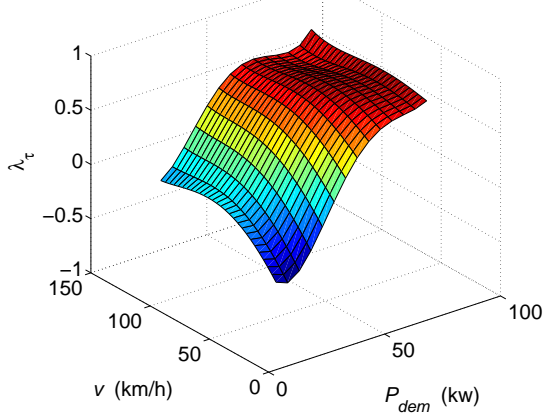

(b)

Figure 7. Decision maps, $\zeta=0.55$. (a) Gear number map; (b) torque split ratio map.

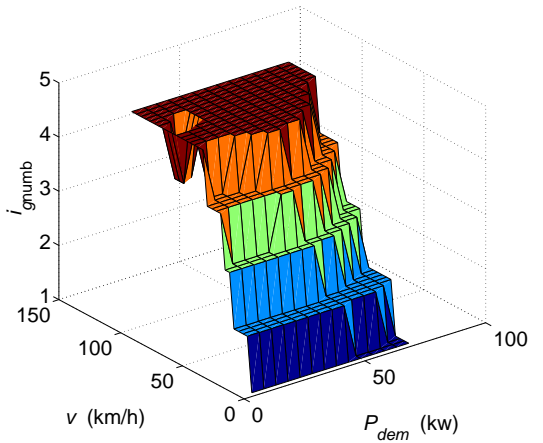

(a)

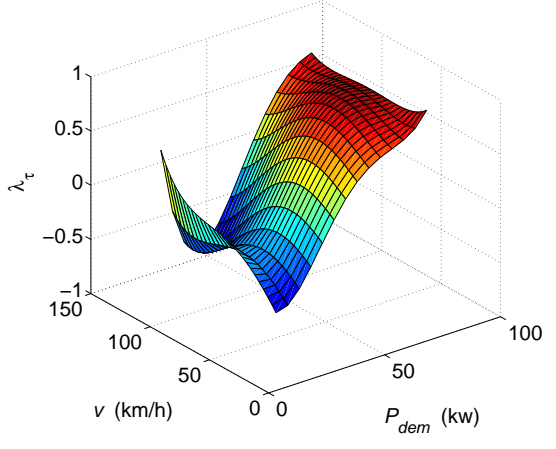

(b)

Figure 8. Decision maps, $\zeta=0.33$. (a) Gear number map; (b) torque split ratio map.

In addition, as shown in the torque split ratio maps (see Figures $6 b, 7 b$ and $8 b$ ), when the energy price ratio is equal to 0.82 , i.e., the electricity is relatively inexpensive, the torque-split ratio mainly concentrates in the region of $\lambda_{\tau}>0$ (see Figure $6 \mathrm{~b}$ ), which means that the electric machine is mainly utilized as the motor to provide the driving torque. However, with the increase of the electricity price, the region of $\lambda_{\tau}>0$ gradually evolved into the region of $\lambda_{\tau}<0$ (see Figures $7 \mathrm{~b}$ and 8b); especially comparing the torque split ratio maps of $\zeta=0.82$ and $\zeta=0.33$, this phenomenon is more obvious. This trend indicates that the electric machine is more often utilized as a generator for saving the cost when the electricity price is increasing. This is mainly because the tradeoff of energy cost between the engine and motor is considered in the power-split scheme. 


\section{Simulation Results and Discussion}

The simulator used for this study is developed in MATLAB and the GT-suite environment. The controller is structured in MATLAB Simulink, and the vehicle model is built in GT-suite. Figure 9 shows the simulation test bench with the GT-Suite simulator.

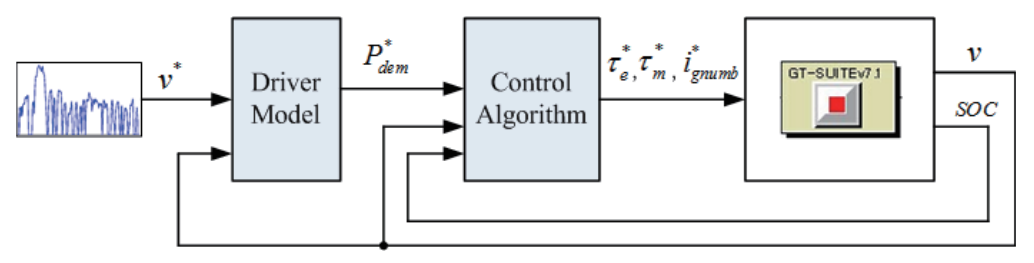

Figure 9. Simulation test bench with the GT-Suite simulator.

In the simulation, the control period, which is consistent with the discrete time step, is selected as $1 \mathrm{~s}$. For each pair of given vehicle speed and power command, the optimal decisions $\lambda_{\tau}$ and $i_{g}$ are solved by using the command fmincon in MATLAB, the corresponding maps are created as shown in Figures 6-8. The vehicle parameters are listed in Table 2. The Urban Dynamometer Driving Schedule (UDDS) cycle is used as the reference vehicle speed profile.

Table 2. Parameters of the vehicle.

\begin{tabular}{cc}
\hline Vehicle Parameters & Value \\
\hline Vehicle mass $m$ & $1500(\mathrm{~kg})$ \\
Radius of the wheel $R_{w}$ & $0.32(\mathrm{~m})$ \\
Air density $\rho$ & $1.16\left(\mathrm{~kg} / \mathrm{m}^{3}\right)$ \\
Drag coefficient $C_{d}$ & $0.3(-)$ \\
Frontal area of vehicle $A$ & $2.5\left(\mathrm{~m}^{2}\right)$ \\
Coefficient of rolling resistance $\mu_{r}$ & $0.01(-)$ \\
Final gear ratio $i_{0}$ & $5.7(-)$ \\
Maximum charge capacity of the battery $Q_{b \max }$ & $32(\mathrm{Ah})$ \\
Open-circuit voltage of the battery $U_{o c}$ & $247(\mathrm{~V})$ \\
Internal resistance of the battery $R_{b}$ & $0.12(\Omega)$ \\
\hline
\end{tabular}

The DP method, as the global optimization method, obviously can obtain better results than the presented map-based method, because the latter is a local optimization method. However, the non-causality of DP algorithm makes it infeasible for real-time control. Therefore, in order to investigate the performance of the proposed HEV power-split strategy, we compare the map-based optimal control (MBOC) approach presented in this study with an applicable nonlinear model predictive control (NMPC) approach presented in the literature [39].

MPC, also known as receding horizon control, is an optimal control strategy based on numerical optimization, in which future control inputs and future plant response are predicted using a system model and optimized at a regular time horizon with respect to a specified cost function. The reason for 
its popularity in industry and academia is that the MPC algorithm can control large systems with many control variables, and most importantly, MPC provides a systematic method of dealing with constraints on inputs and states.

The developed NMPC algorithm provides an on-line solution of the formulated nonlinear energy management optimal control problem. Due to the nonlinearities in the system dynamics and the physical constraints, a general numerical method for MPC, the continuous/GMRES (generalized minimum residual)-based method, is employed to solve the control problem. Figure 10 shows a schematic diagram of the NMPC algorithm. According to the current vehicle states $v(t)$ and $S O C(t)$, as well as the current external inputs in terms of the driver demand power $P_{d e m}(t)$ and the road slopes information $\theta(t)$, the NMPC algorithm can give the sequences of optimal control variables $\left(\lambda_{\tau t}(i)\right.$ and $i_{g t}(i)$, $i=0,1, \cdots, N-1)$ during the predictive horizon $T$ by solving a linear differential equation in real time. This differential equation is with respect to the necessary conditions of Pontryagin's minimum principle. Then, the $\lambda_{\tau t}(0)$ and $i_{g t}(0)$ are implemented as the control inputs. For more details on this NMPC method, refer to [39].

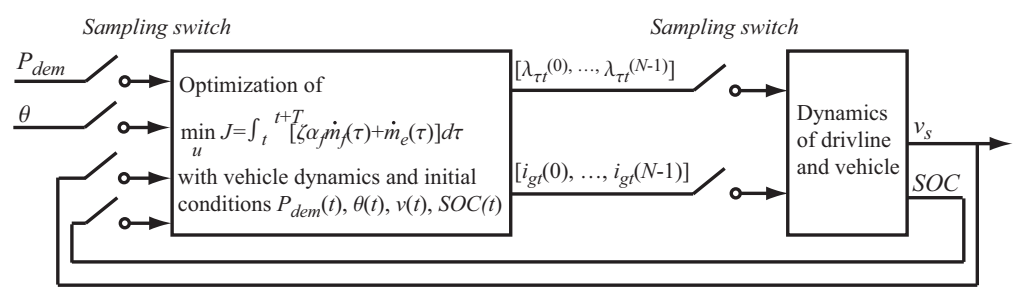

Figure 10. Schematic diagram of the nonlinear MPC (NMPC) algorithm.

For the MBOC algorithm, due to the decisions being extracted from the static maps, the extraction time for every decision thus can be regarded as instantaneous. However, for the NMPC algorithm, the on-line computation time for every decision is about $20 \mathrm{~ms}$ under the main configuration of the computer: Intel Dual-core CPU $1.7 \mathrm{GHz} \times 2$, memory $(\mathrm{RAM}) 4 \mathrm{~GB}$. Therefore, it is illustrated that the computational load can be significantly decreased by the proposed MBOC algorithm. The other comparison results between the MBOC algorithm and NMPC algorithm are shown as follows.

Figure 11 shows the fuel, electricity and total cost (the sum of electricity and fuel cost) under an appointed energy price ratio $\zeta=0.82$ over this drive cycle. It can be observed that the MBOC strategy achieves less total energy cost for this trip compared to the NMPC strategy, despite the fuel cost being more. This benefit is evidently attributed to the greater savings of the electricity cost. Owing to less electricity energy being consumed in the MBOC strategy, the SOC therefore retains a higher value (0.757) at the end of the trip than that (0.638) in NMPC, as seen in Figure 12.

For different energy price ratios, a quantitative comparison on energy cost between these two control strategies is reported in Table 3. In this table, the positive sign in the term of improvement represents the reduction of the energy cost, and the negative sign represents the increase of that. From this table, it can be noted that the fuel cost by the MBOC strategy is increased by $16.9 \%, 11.5 \%$ and $11.2 \%$ compared to that by the NMPC strategy under energy price ratios of $\zeta=0.82, \zeta=0.55$ and $\zeta=0.33$, respectively; the electricity costs are significantly decreased by $45.8 \%, 34.7 \%$ and $55.1 \%$ for the MBOC strategy over the NMPC strategy, respectively. Therefore, the total costs are accordingly saved by $6.5 \%, 7.7 \%$ 
and $17.0 \%$, respectively. Although these two policies, as local optimization methods, both will obtain approximate optimal solutions, the improvement on the total cost indicates that the decision obtained by the MBOC method is more optimal than that obtained by the NMPC method. This is mainly because, on the one hand, the accuracy of the discrete model directly affects the accuracy of the decision in each time horizon, consequently influencing the energy consumption. For the MBOC strategy proposed in this study, the vehicle model is discretized by the discrete variational method; this discrete model is more accurate than the common discrete method used in NMPC under the same time step. On the other hand, as a real-time predictive method, the on-line NMPC is sensitive to transient disturbance and noise, which often leads to the overaction. However, the proposed offline MBOC is calmer when making the decision; the energy consumption thus is improved.

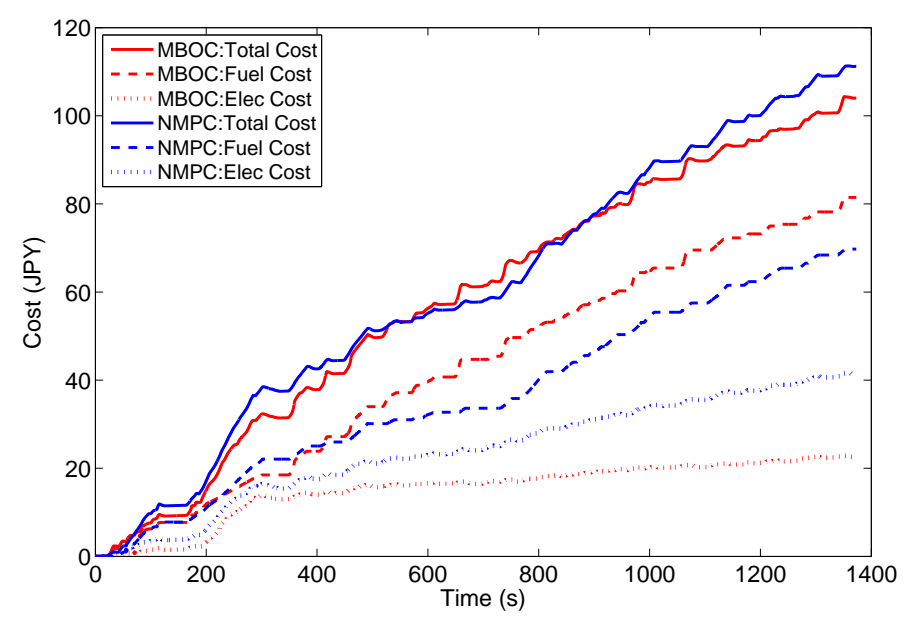

Figure 11. Cost for MBOC and NMPC strategies in $\zeta=0.82$.

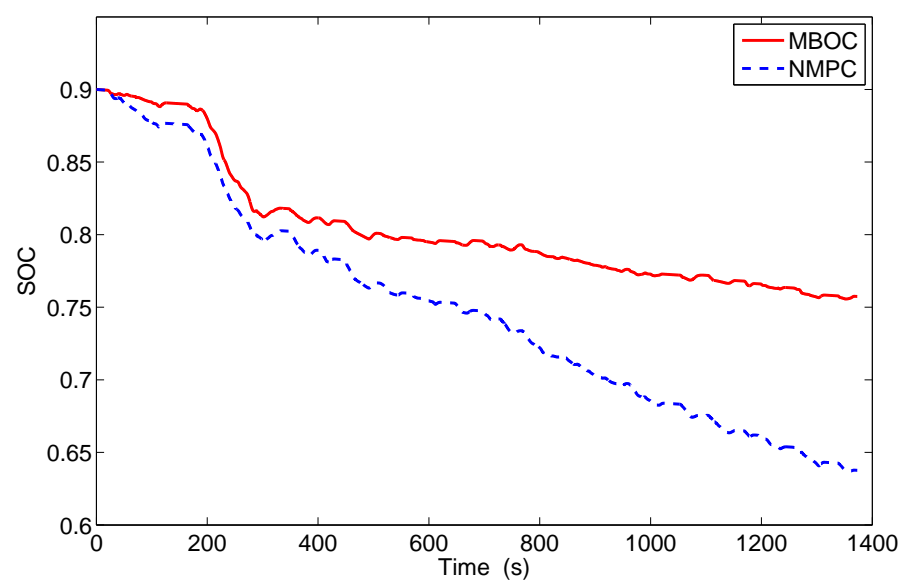

Figure 12. SOC for MBOC and NMPC strategies in $\zeta=0.82$. 
Table 3. Comparison of energy cost for MBOC and NMPC strategies in $\zeta=0.82, \zeta=0.55$ and $\zeta=0.33$.

\begin{tabular}{llllllllll}
\hline & \multicolumn{4}{c}{$\zeta=\mathbf{0 . 8 2}$} & \multicolumn{3}{c}{$\zeta=\mathbf{0 . 5 5}$} & \multicolumn{3}{c}{$\zeta=\mathbf{0 . 3 3}$} \\
Controller & MBOC & NMPC & Improvement & MBOC & NMPC & Improvement & MBOC & NMPC & Improvement \\
\hline Fuel Cost (JPY) & 81.5 & 69.7 & $-16.9 \%$ & 78.6 & 70.5 & $-11.5 \%$ & 97.4 & 87.6 & $-11.2 \%$ \\
Electricity Cost (JPY) & 22.5 & 41.5 & $+45.8 \%$ & 32.8 & 50.2 & $+34.7 \%$ & 29.2 & 65.0 & $+55.1 \%$ \\
Total Cost (JPY) & 104.0 & 111.2 & $+6.5 \%$ & 111.4 & 120.7 & $+7.7 \%$ & 126.6 & 152.6 & $+17.0 \%$ \\
\hline
\end{tabular}

A remarkable characteristic of the presented power management approach is that the optimal decisions strongly depends on the energy price ratio $\zeta$. In order to research the property of this dependence, we adopt three different energy price ratios as selected above in the MBOC approach and give the results of energy consumption over this drive cycle as listed in Table 4. Moreover, the detailed comparison on the operational characteristics of the vehicle between the energy price ratio $\zeta=0.82$ and $\zeta=0.33$ are given in Figures 13 to 15 .

Table 4. Comparison of the energy cost for the MBOC strategy in $\zeta=0.82, \zeta=0.55$ and $\zeta=0.33$.

\begin{tabular}{lccc}
\hline Energy Price Ratio & $\zeta=\mathbf{0 . 8 2}$ & $\zeta=\mathbf{0 . 5 5}$ & $\zeta=\mathbf{0 . 3 3}$ \\
\hline Fuel consumption (g) & 407.4 & 393.0 & 487.0 \\
Electricity consumption (kWh) & 1.127 & 1.093 & 0.583 \\
Total consumption (MJ) & 18.72 & 13.37 & 9.11 \\
Terminal SOC (-) & 0.757 & 0.762 & 0.826 \\
\hline
\end{tabular}
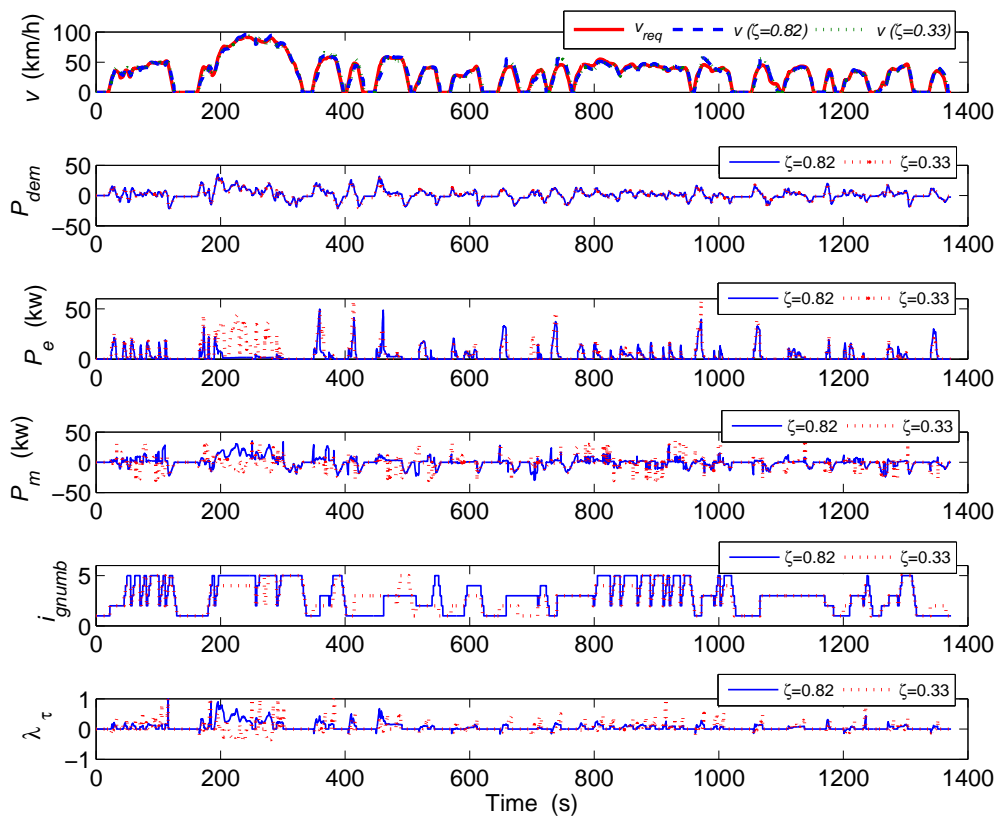

Figure 13. Vehicle speed, power demand, engine power, motor power, gear number and torque split ratio: $\zeta=0.82 \mathrm{vs} . \zeta=0.33$. 
Table 4 shows that the electricity consumption drops from 1.127 to 0.583 kilo-watt hour, and the terminal SOC of the battery accordingly changes from 0.757 to 0.826 for this trip when the energy price ratio varies from 0.82 to 0.33 . This trend agrees with the fact that the electricity energy should be less and less used with the increase of electricity price. The total consumption, which is calculated by converting the fuel consumption in grams to joules and the electricity consumption in kilo-watt hours to joules and then summing them in the same units, reduces from 18.72 to 9.11 megajoules; namely, about half of the energy is saved. The reason will be explained in Figure 15. As a matter of fact, the total consumption given here, as the real energy consumption of the engine and motor, is not equivalent to the selected cost function, which is the weighted energy consumption and equivalent to the energy cost. Therefore, for the same fuel price and driving condition, the rise of electricity price will inevitably lead to the increase of the total cost, despite the actual consumption being less. This fact can be illustrated in the data of the total cost in Table 3.

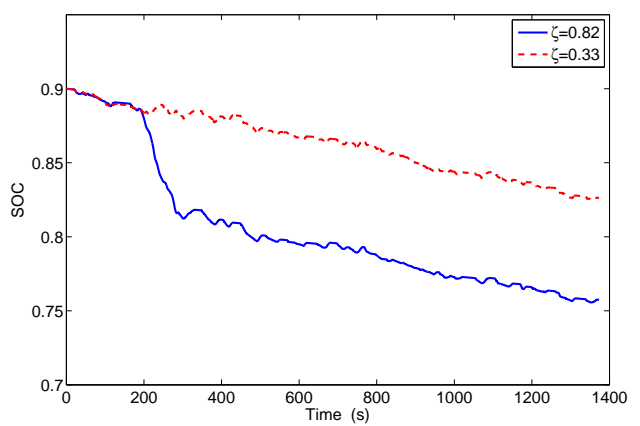

Figure 14. Battery SOC: $\zeta=0.82 v s . \zeta=0.33$.

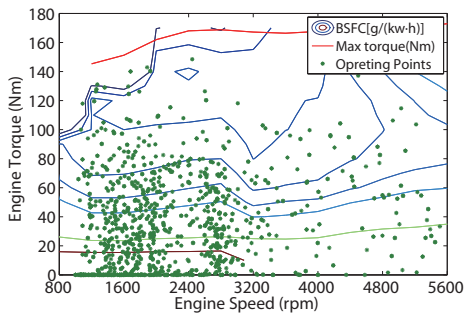

(a)

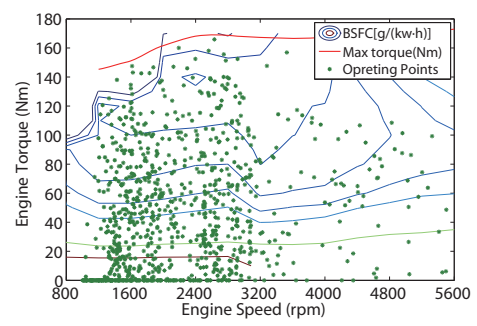

(c)

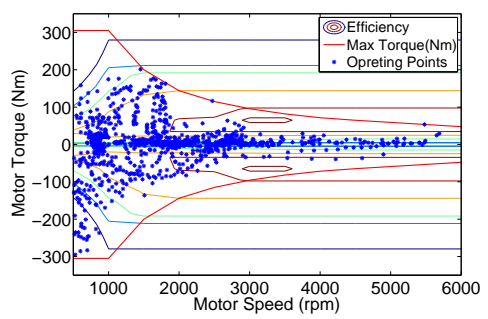

(b)

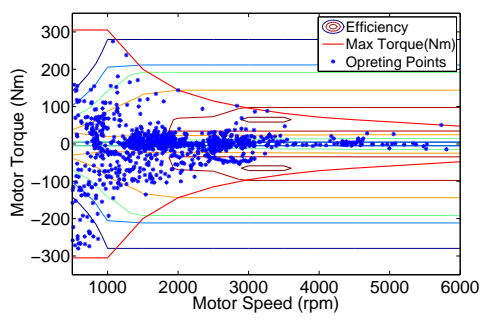

(d)

Figure 15. Operating points of the engine and motor in $\zeta=0.82$ and $\zeta=0.33$. (a) Operating points of the engine, $\zeta=0.82$; (b) operating points of the motor, $\zeta=0.82$; (c) operating points of the engine, $\zeta=0.33 ;(\mathbf{d})$ operating points of the motor, $\zeta=0.33$. 
From Figure 13, it is observed that the MBOC strategy can track the speed profile successfully; the power demand is almost the same under the two different parameters of the energy price ratio. Since the different energy price ratio leads to different decisions, as given in Figures 6 and 8, the power distributed to the engine and motor thus is different during this trip. With the reduction of the electricity price, more electricity power is consumed (see Figure 14). Especially in a high speed situation, for example from around the 200th to 300th second, the power demand in $\zeta=0.33$ mainly is met by the engine, and the power provided by the engine is more than the power demand; the excess part of the engine power is used to charge the battery. In contrast, in $\zeta=0.82$, the motor provides the main power demand in the same section of the speed profile, the SOC of the battery, thus has a rapid decline, as shown in Figure 14.

Figure 15 presents the operating points of the engine and motor for two energy price ratios of $\zeta=0.82$ and $\zeta=0.33$. By comparing Figure 15a to Figure 15c, it can be found that more operating points of the engine, in rotation speed ranging from 1000 to $3000 \mathrm{rpm}$, distribute to the region where the BSFC value is lower than $260 \mathrm{~g} / \mathrm{kWh}$. It also can be noticed from Figure 15b,d that more operating points of the motor, in the same rotation speed range, concentrate on the region where the efficiency is larger than 85 percent. These phenomenon indicate that, for a low energy price ratio (i.e., fuel is relatively inexpensive compared to electricity), the proposed power management strategy makes the engine and motor work with high efficiency; therefore, the real energy consumption is significantly reduced, as reported in Table 4. However, from the viewpoint of economic cost, as is the concern of the current study, some expenditures have been saved under an inexpensive electricity price, even though this strategy forces the actuators to run with relatively low efficiency.

\section{Conclusions}

In the last two decades, much attention for the design of the HEV energy management strategy has focused on the optimization problem, expect for the rule-based control strategy proposed at an early stage. Nevertheless, most of the optimization-based strategies, especially the dynamical programming algorithm, require the targeted driving route to be known previously and to generate heavy computational loads in the optimizing process. Recently, a few literature works challenged the real-time optimization problem using receding horizon optimal control or model-based predictive control [26,39]; however, these methods solved the approximate solution in exchange for reducing the on-line computational load.

This paper challenged this tradeoff by using a map-based approach. It should be noted that the outputs of static maps, which provide the torque split and gear shift commands, are not only current state-dependent decisions, but also optimal decisions with respect to the vehicle dynamics and model-based predicted transient performance. Furthermore, the maps are generated by off-line calculation with a discrete time dynamical model of targeted vehicles that is obtained by a discrete variational method. The effectiveness of the proposed approach is validated with the GT-Suit simulator, which is widely used in the automotive industry, and the comparison with the real-time optimization algorithm shows that, for a specified UDDS cycle, the total costs are significantly decreased by $6.5 \%$, $7.7 \%$ and $17.0 \%$ for the proposed map-based optimal control strategy over the on-line nonlinear MPC strategy under different energy price ratios of $0.82,0.55$ and 0.33 . Finally, it is noticed that the dynamics of the battery SOC is not included in the dynamical constraint for the optimization, due to the long-term 
dynamics of the battery SOC compared to the vehicle dynamic, and for the sake of the simplicity of the optimization algorithm. In the design of optimal power-split maps, a comprehensive consideration including the influence of battery SOC will be further work.

\section{Conflicts of Interest}

The authors declare no conflict of interest.

\section{Abbreviations}

EV Electric vehicle

FCEV Fuel cell electric vehicle

HEV Hybrid electric vehicle

PHEV Plug-in hybrid electric vehicle

DP Dynamical programming

SOC State of charge

MPC Model predictive control

EM Electric machine

ICE Internal combustion engine

BR Battery recover

BSFC Break specific fuel consumption

SQP Sequential quadratic programming

QP Quadratic programming

UDDS Urban dynamometer driving schedule

NMPC Nonlinear model predictive control

MBOC Map-based optimal control

GMRES Generalized minimum residual

\section{References}

1. Katrašnik, T. Hybridization of powertrain and downsizing of IC engine-A way to reduce fuel consumption and pollutant emission-Part I. Energy Convers. Mang. 2007, 48, 1411-1423.

2. Matthé, R.; Eberle, U. The voltec system-energy storage and electric propulsion. In Lithium-Ion Batteries; Pistoia, G., Ed.; Elsevier: Amsterdam, The Netherlands, 2014; pp. 151-176.

3. Tanoue, K.; Yanagihara, H.; Kusumi, H. Hybrid is a key technology for future automobiles. In Hydrogen Technology; Léon, A., Ed.; Springer: Berlin, Germany, 2008; pp. 235-272.

4. Stephen, C.H.; Sullivan, J. Environmental and energy implications of plug-in hybrid-electric vehicle. Environ. Sci. Technol. 2008, 42, 1185-1190.

5. Chau, K.T.; Wong, Y.S. Overview of power management in hybrid electric vehicles. Energy Convers. Mang. 2002, 43, 1953-1968.

6. Wirasingha, S.G.; Emadi, A. Classification and review of control strategies for plug-in hybrid electric vehicle. IEEE Trans. Veh. Technol. 2011, 60, 111-122. 
7. Serrao, L.; Onori, S.; Rizzoni, G. A comparative analysis of energy management strategies for hybrid electric vehicles. J. Dyn. Syst. Meas. Control 2011, 133, 1-9.

8. Banvait, H.; Anwar, S.; Chen, Y. A rule-based energy management strategy for plug-in hybrid electric vehicle(PHEV). In Proceedings of the American Control Conference, St. Louis, MO, USA, 10-12 June 2009.

9. Abdelsalam, A.A.; Cui, S. A fuzzy logic global power management strategy for hybrid electric vehicles based on a permanent magnet electric variable transmission. Energies 2012, 80, 1175-1198.

10. Trovão, J.P.; Pereirinha, P.G.; Jorge, H.M.; Antunes, C.H. A multi-level energy management system for multi-source electric vehicles-An integrated rule-based meta-heuristic approach. Appl. Energy 2013, 105, 304-318.

11. Jalil, N.; Kheir, N.A.; Salman, M. Rule-based energy management strategy for a series hybrid vehicle. In Proceedings of the American Control Conference, Albuquerque, MI, USA, 6 June 1997.

12. Schouten, N.J.; Salman, M.A.; Kheir, N.A. Fuzzy logic control for parallel hybrid vehicles. IEEE Trans. Control Syst. Technol. 2002, 10, 460-468.

13. Sharer, P.B.; Rousseau, A.; Karbowski, D.; Pagerit, S. Plug-in hybrid electric vehicle control strategy: Comparison between EV and charge-depleting options. Technical Paper 2008-01-0460. In Proceedings of the SAE, Detroit, MI, USA, 14-17 April 2008.

14. Pisu, P; Rizzoni, G. A comparative study of supervisory control strategies for hybrid electric vehicles. IEEE Trans. Control Syst. Technol. 2007, 15, 506-518.

15. Nüesch, T.; Elbert, P.; Flankl, M.; Onder, C.; Guzzella, L. Convex optimization for the energy management of hybrid electric vehicles considering engine start and gearshift costs. Energies 2014, 7, 834-856.

16. Hou, C.; Ouyang, M.; Xu, L.; Wang, H. Approximate pontryagin's minimum principle applied to the energy management of plug-in hybrid electric vehicles. Appl. Energy 2014, 115, 174-189.

17. Larsson, V.; Johannesson Mårdh, L.; Egardt, B.; Karlsson, S. Commuter route optimized energy management of hybrid electric vehicles. IEEE Trans. Intell. Transp. Syst. 2014, 15, 1145-1154.

18. Lin, C.C.; Peng, H.; Grizzle, J.W.; Kang, J.M. Power management strategy for a parallel hybrid electric truck. IEEE Trans. Control Syst. Technol. 2003, 11, 839-849.

19. Moura, S.J.; Fathy, H.K.; Callaway, D.S.; Stein, J.L. A stochastic optimal control approach for power management in plug-in hybrid electric vehicles. IEEE Trans. Control Syst. Technol. 2011, $19,545-555$.

20. Gong, Q.; Li, Y.; Peng, Z.R. Trip-based optimal power management of plug-in hybrid electric vehicles. IEEE Trans. Veh. Technol. 2008, 57, 3393-3401.

21. Scordia, J.; Desbois-Renaudin, M.; Trigui, R.; Jeanneret, B.; Badin, F.; Plasse, C. Global optimization of energy management laws in hybrid vehicles using dynamic programming. Int. J. Veh. Des. 2005, 39, 349-367.

22. Moura, S.J.; Callaway, D.S.; Fathy, H.K.; Stein, J.L. Tradeoffs between battery energy capacity and stochastic optimal power management in plug-in hybrid electric vehicles. J. Power Sources 2010 , 195, 2979-2988.

23. Sciarretta, A.; Guzzella, L. Control of hybrid electric vehicles. IEEE Control Syst. Mag. 2007, 27, 60-70. 
24. Sundstrom, O.; Guzzella, L.; Soltic, P. Torque-assist hybrid electric powertrain sizing: from optimal control towards a sizing law. IEEE Trans. Control Syst. Technol. 2010, 18, 837-849.

25. Tulpule, P.; Marano, V.; Rizzoni, G. Effect of traffic, road and weather information on phev energy management. Technical Paper 2011-24-0162. In Proceedings of the SAE, Detroit, MI, USA, 12-14 April 2011.

26. Borhan, H.; Vahidi, A.; Phillips, A.M.; Kuang, M.L.; Kolmanovsky, I.V.; di Cairano, S. MPC-based energy management of a power-split hybrid electric vehicle. IEEE Trans. Control Syst. Technol. 2012, 20, 593-603.

27. Yan, F.; Wang, J.; Huang, K. Hybrid electric vehicle model predictive control torque-split strategy incorporating engine transient characteristic. IEEE Trans. Veh. Technol. 2012, 61, 2458-2467.

28. Zhang, J.Y.; Shen, T.L. Nonlinear MPC-based power-assist scheme of internal combustion engines in plug-in hybrid electric vehicles. In Proceedings of the European Control Conference, Strasbourg, France, 24-27 June 2014.

29. Bemporad, A.; Morari, M.; Dua, V.; Pistikopoulos, E.N. The explicit linear quadratic regulator for constrained systems. Automatica 2002, 38, 3-20.

30. Bemporad, A.; Borrelli, F.; Morari, M. Model predictive control based on linear programming-The explicit solution. IEEE Trans. Automat. Control 2002, 47, 1974-1985.

31. Ohtsuka, T. A continuation/GMRES method for fast computation of nonlinear receding horizon control. Automatica 2004, 40, 563-574.

32. Richter, S.; Jones, C.N.; Morari, M. Computational complexity certification for real-time MPC with input constraints based on the fast gradient method. IEEE Trans. Automat. Control 2012, 57, 1391-1403.

33. Marsden, J.E.; West, M. Discrete mechanics and variational integrators. Acta Numer. 2011, 10, 357-514.

34. Chyba, M.; Hairer, E.; Vilmart, G. The role of symplectic intergrators in optimal control. Optim. Control Appl. Methods 2009, 30, 367-382.

35. Van Mierlo, J.; Van den Bossche, P.; Maggetto, G. Models of energy sources for EV and HEV: fuel cells, batteries, ultracapacitors, flywheels and engine-generators. J. Power Sources 2004, 128, 76-89.

36. Rizzoni, G.; Guzzella, L.; Baumann, B.M. Unified modeling of hybrid electric vehicle drivetrains. IEEE-ASME Trans. Mech. 1999, 4, 246-257.

37. Gill, P.E.; Murray, W.; Wright, M.H. Practical Optimization; Academic Press: London, UK, 1982.

38. Boggs, P.T.; Tolle, J.W. Sequential Quadratic Programming. Acta Numer. 1995, 4, 1-51.

39. Zhang, J.Y.; Shen, T.L.; Sawada, T.; Kubo, M. Nonlinear MPC-based power management strategy for plug-in parallel hybrid electrical vehicles. In Proceedings of the Chinese Control Conference, Nanjing, China, 28-30 July 2014.

(c) 2015 by the authors; licensee MDPI, Basel, Switzerland. This article is an open access article distributed under the terms and conditions of the Creative Commons Attribution license (http://creativecommons.org/licenses/by/4.0/). 